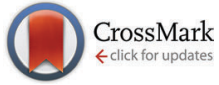

Cite this: Phys. Chem. Chem. Phys., 2015, 17, 10579

Received 30th January 2015, Accepted 7th March 2015

DOI: $10.1039 / \mathrm{c} 5 \mathrm{cp} 00609 \mathrm{k}$

www.rsc.org/pccp

\section{Modulated excitation extended X-ray absorption fine structure spectroscopy $\dagger$}

\begin{abstract}
Gian Luca Chiarello*ab and Davide Ferri ${ }^{c}$
The sensitivity of extended X-ray absorption fine structure spectroscopy (EXAFS) for minute structural changes can be enhanced by combination with the modulated excitation approach and making use of phase sensitive analysis. A modulated EXAFS experiment of a reversible periodic $\mathrm{Pd}$ to $\mathrm{PdO}$ partial oxidation has been simulated in order to understand the effect of the phase sensitive analysis on the shape and meaning of the resulting phase-resolved EXAFS spectra. In particular, the simulation comprises either a synchronous or a delayed sinusoidal variation of the EXAFS parameters, i.e. coordination number $(N)$, interatomic distance $(R)$ and Debye-Waller factor $\left(\sigma^{2}\right)$, of first $\mathrm{Pd}-\mathrm{Pd}$, first $\mathrm{Pd}-\mathrm{O}$, and second $\mathrm{Pd}-(\mathrm{O})-\mathrm{Pd}$ coordination shells. The effect of these variations on the resulting phase-resolved Fourier transform EXAFS spectra is discussed. The results of the simulation are validated by an in situ EXAFS experiment at the $\mathrm{Pd} \mathrm{K}$-edge over 1.6 wt\% $\mathrm{Pd} / \mathrm{Al}_{2} \mathrm{O}_{3}$ undergoing reversible partial oxidation in a $\mathrm{H}_{2}$ vs. $\mathrm{O}_{2}$ modulation at $573 \mathrm{~K}$. It is shown that phase sensitive detection (PSD) is able to separate the minor contribution at ca. $2.8 \AA$ corresponding to the growth of the $\mathrm{Pd}-(\mathrm{O})-\mathrm{Pd}$ shell that is otherwise hidden under the static signal of the $\mathrm{Pd}-\mathrm{Pd}$ shell of reduced Pd particles. The fitting of the phase-resolved EXAFS spectra suggests that the fast $\mathrm{H}_{2}$ to $\mathrm{O}_{2}$ switch leads the partial oxidation of the Pd surface with the formation of a PdO shell covering a metallic Pd core. Therefore, the dynamics of the full system can be described with greater detail than in conventional EXAFS. The intention of this work is to provide the tools and therefore a solid guidance to qualitatively and quantitatively understand the nature of the shape of phase-resolved FT-EXAFS spectra that may prove helpful in the analysis of a wide range of functional materials.
\end{abstract}

\section{Introduction}

The structure of the active surface species, e.g. the nature and geometry of adsorbates and of coordination sites on metal atoms, is a central issue to heterogeneous catalysis. X-ray Absorption Spectroscopy (XAS) has been largely employed in this context in an attempt to solve the oxidation state and the element specific local structure of the active sites, for example, on supported metal nano-particles while the catalytic event takes place. ${ }^{1}$ A vast improvement of knowledge on the structure of catalysts under working conditions has been achieved with both temporal and spatial resolution. However, XAS is intrinsically limited to bulk information due to the high penetration depth of the X-rays. Moreover, structural conclusions are usually derived from experiments performed under steady state conditions.

\footnotetext{
${ }^{a}$ Università degli Studi di Milano, Dipartimento di Chimica, Via C. Golgi 19, I-20133 Milano, Italy. E-mail: gianluca.chiarello@unimi.it; Fax: +3902 503 14300; Tel: +3902 50314281

${ }^{b}$ Empa, Swiss Federal Laboratories for Materials Science and Technology, Ueberlandstrasse 129, CH-8600 Dübendorf, Switzerland

${ }^{c}$ Paul Scherrer Institut, $\mathrm{CH}-5232$ Villigen PSI, Switzerland

$\dagger$ Electronic supplementary information (ESI) available. See DOI: 10.1039/ c5cp00609k
}

Therefore, it may be difficult to ultimately discern the underlying red-ox dynamics of the catalyst in the catalytic cycle. Perturbation of the catalyst via modification of an external parameter can be used to enhance the catalyst performance ${ }^{2}$ and even more central to the spectroscopic investigations, it serves as an analytical expedient to obtain a deeper insight into reaction mechanisms. In this respect, Modulated Excitation Spectroscopy (MES), ${ }^{3-5}$ achieved through the periodic variation of the gas composition of feedstock, has been recently combined with time-resolved XAS. ${ }^{6-10}$ The combination revealed a powerful tool to convey enhanced sensitivity to XAS. MES exploits Phase Sensitive Detection (PSD) to increase the signal to noise ratio and to remove those parts of the spectra that are not affected by the external stimulation (e.g. the periodic change of the feedstock composition), significantly improving the active species response. This approach becomes particularly important for unveiling structural information of working nano-particles. Depending on the particle size, only a small fraction of atoms is usually involved in adsorption/reaction processes while their spectroscopic signature is often characterized by weak signals and hidden by the dominant static signals, e.g. of the nano-particle bulk. In the case of small particles of relevant (low) metal loadings of complex catalysts the noise will largely dominate the EXAFS signal. 
For metal oxide based catalysts, similar sensitivity improvements are anticipated.

PSD is a function that transforms a set of time-resolved data $I(t)$, with $0<t<T$ ( $T$ being the stimulation period), into a new set of phase-resolved data $I\left(\varphi^{\mathrm{PSD}}\right)$, with $0^{\circ}<\varphi^{\mathrm{PSD}}<360^{\circ}\left(\varphi^{\mathrm{PSD}}\right.$ is the demodulation phase angle), according to the following equation:

$$
I\left(\varphi^{\mathrm{PSD}}\right)=\frac{2}{T} \int_{0}^{T} I(t) \sin \left(K \omega t+\varphi^{\mathrm{PSD}}\right) \mathrm{d} t
$$

where $\omega(2 \pi / T)$ is the stimulation frequency and $K$ is the demodulation index ( $K=1$ is the fundamental harmonics). The use of MES in combination with XAS has been inspired by and borrowed from the combination of MES and IR spectroscopy. ${ }^{4,5,11}$ In this case, the periodic switch of gas composition affects the concentration of the surface active species with the consequent periodic changes of their characteristic absorption in the IR spectrum.

In previous reports considering supported $\mathrm{Pd}$ and $\mathrm{Rh}$ nanoparticles, ${ }^{6-8,12,13}$ PSD was applied directly to the time-resolved XAS spectra. Though the method was demonstrated to be powerful for massively enhancing the EXAFS oscillations against the static response, a qualitative interpretation only of the region around the edge, the whiteline and $c a$. 150-200 eV above it was carried out. ${ }^{7,8,12}$ The result is that the ME-XAS is a valid alternative to the differential $\Delta \mu$-XANES technique ${ }^{14,15}$ with the advantage of the higher signal to noise ratio and its intrinsic in situ nature (and no need for reference spectra in a vacuum). Contrary to the case of Rh where the catalyst structure oscillates between two boundary states, i.e. oxidized and reduced $\mathrm{Rh}$, apparently without additional species, the phaseresolved spectra of $\mathrm{Pd} / \mathrm{Al}_{2} \mathrm{O}_{3}$ exhibited a deviation from this behaviour. This was associated with the formation of $\mathrm{Pd}$ carbide species according to conventional EXAFS data fit. ${ }^{8}$ König et $a l .{ }^{9}$ provided the first quantitative evaluation of phase-resolved EXAFS data after following the same procedure, thus demonstrating the great potential of this combination for elucidation of structural variations during reaction. Changes of coordination number and bond distances as low as $1 \%$ and $0.001 \AA$ could be detected, respectively. The procedure for the isolation of transient structures additional to the two boundary states (oxidized and reduced metal) from phase-resolved EXAFS data was recently described by the same group. ${ }^{16}$ The massive enhancement of sensitivity provided by the method was further verified with the detection of sulfur containing species on $\mathrm{Ru}$ nano-particles while Ru changed from being partially oxidized to reduced.

Recently, Stötzel et al. have raised some doubts about the advantage of coupling ME with EXAFS followed by PSD data treatment. ${ }^{17}$ Thus, a more careful description of the possibilities at hand for application of PSD to XAS datasets is required even on a qualitative base. This has also been stimulated by the theoretical accounts of ME-XRD. ${ }^{18-20}$ As in the case of XRD, the interpretation of an EXAFS spectrum in the phase domain might turn into a more complex task than in the case of vibrational spectroscopy. During a modulation experiment, the periodic switch of e.g. gas composition affects the local structure around the absorber atom. Given the nature of the X-ray absorption process and thus of the EXAFS spectrum a greater number of variables are involved. The EXAFS spectrum in the $k$ domain $\chi(k)$ is described by the well known equation, ${ }^{21}$

$$
\chi(k)=\sum_{i} \frac{\left(N_{i} S_{0}^{2}\right) F_{i}(k)}{k R_{i}^{2}} \sin \left[2 k R_{i}+\delta_{i}(k)\right] \mathrm{e}^{-2 \sigma_{i}{ }^{2} k^{2}} \mathrm{e}^{-\frac{2 R_{i}}{\lambda(k)}}
$$

where the summation is extended to all of the possible $i$ photoelectron scattering paths. The terms $F_{i}(k), \delta_{i}(k)$, and $\lambda(k)$ are the scattering amplitude, the phase shift, and the mean free path of the photoelectron, respectively. Because these terms are scattering properties that depend only on the nature of the neighboring atoms of the excited atom, they are not affected by the modulation experiment. This applies also to $S_{0}{ }^{2}$, the passive electron reduction factor. In contrast, the terms $N_{i}$ (degeneracy of identical paths), $R_{i}$ (half path length) and $\sigma_{i}^{2}$ (Debye-Waller factor) of eqn (2) are structural parameters and can vary as a consequence of the modulation. Thus, a phase domain EXAFS spectrum can depend on up to a maximum of $3 n$ variables, $n$ being the number of paths affected by the stimulation.

As a complement to the previous reports where the XANES region was specifically treated,,$^{7,8,12,22}$ we proceed here to perform the phase sensitive analysis of time-resolved EXAFS data in the $k$ space and discuss the effects of the variation of the structural parameters $\left(N, R\right.$ and $\left.\sigma^{2}\right)$ on the shape and intensity of observable changes in the corresponding Fourier transform data set. In order to facilitate the interpretation of phase domain FT-EXAFS data, we will first analyze simulated EXAFS spectra at the Pd K-edge of an ideal Pd surface that undergoes reversible partial re-oxidation. This approach is not intended to simulate the real red-ox dynamics of catalytic Pd nano-particles. More sophisticated DFT calculations are necessary for this purpose. The aim of this simulation is rather the systematic evaluation of the effect of a periodic variation of the structural parameters on the shape of phase-resolved spectra. The effect of the variation of the three structural parameters on a single coordination shell or on different shells will be treated separately. Finally, the results of the simulation will be verified on a relatively simple experimental system for catalysis consisting of $\mathrm{Pd}$ nano-particles supported on $\mathrm{Al}_{2} \mathrm{O}_{3}$ subjected to reduction and re-oxidation cycles.

\section{Experimental}

\subsection{Simulation of EXAFS spectra}

In order to explore the reliability of the application of the PSD algorithm to EXAFS spectra a set of simulated time-resolved spectra at the Pd K-edge has been considered first. These spectra have been generated considering a metallic Pd surface subjected to a modulation experiment where the gas atmosphere is periodically switched from reducing (e.g. $\mathrm{H}_{2}$-rich) to oxidizing (e.g. $\mathrm{O}_{2}$-rich) with a full modulation period $(T)$ of 100 s. Under these conditions, we can suppose that $\mathrm{Pd}$ is periodically partially oxidized in the presence of $\mathrm{O}_{2}$ and then reduced in the following $\mathrm{H}_{2}$-rich pulse. In a first approximation, the metal state can be described by considering only the first 
Pd-Pd scattering shell, whereas the oxidized state is well represented by both the first $\mathrm{Pd}-\mathrm{O}$ and the second $\mathrm{Pd}-(\mathrm{O})-\mathrm{Pd}$ scattering shells. Considering that the same number of atoms takes part to the periodic oxidation-reduction process (absence of disaggregation and re-construction), ${ }^{23}$ the oxidation proceeds reasonably from the surface to the bulk, thus provoking the decrease of the average coordination number of the Pd-Pd shell (this is particularly true in the case of Pd nanoparticles) and the simultaneous appearance of the $\mathrm{Pd}-\mathrm{O}$ and $\mathrm{Pd}-(\mathrm{O})-\mathrm{Pd}$ shells.

The simulated EXAFS spectra at the Pd K-edge have been obtained using the scattering paths generated with the FEFF6 code. ${ }^{24}$ The structural parameters $\left(N, R\right.$ and $\left.\sigma^{2}\right)$ of the three considered scattering paths (Pd-Pd, $\mathrm{Pd}-\mathrm{O}$ and $\mathrm{Pd}-(\mathrm{O})-\mathrm{Pd})$ were varied as a sinusoidal function of the frequency $\omega$, equal to the simulated modulation frequency $(\omega=2 \pi / T)$. All simulated and experimental spectra have been analyzed and processed using the IFFEFIT software package, ${ }^{25}$ including Athena for data normalization, background subtraction, $k$-weighting and Fourier transformation and Artemis for data fitting. All reported FT-EXAFS spectra are not phase corrected.

\subsection{Modulated excitation quick-EXAFS experiment}

Time-resolved quick-EXAFS spectra were collected at the Pd K-edge $(24.35 \mathrm{keV})$ at the beamline BM23 of ESRF (Grenoble, France). The $1.6 \mathrm{wt} \% \mathrm{Pd} / \mathrm{Al}_{2} \mathrm{O}_{3}$ sample $\left(141 \mathrm{~m}^{2} \mathrm{~g}^{-1} ; 13 \% \mathrm{Pd}\right.$ dispersion; Pd particles size of $c a .6 \mathrm{~nm}$ ), kindly provided by Umicore, was tested using an in situ reactor cell designed for simultaneous XAS/DRIFTS measurements, ${ }^{26}$ though no DRIFT spectra have been considered for this work. The outlet gas composition was constantly monitored using mass spectrometry (MS). ${ }^{26,27}$ The sample was heated to $573 \mathrm{~K}$ under flowing $1 \mathrm{vol} \% \mathrm{O}_{2} / \mathrm{He}\left(80 \mathrm{ml} \mathrm{min}^{-1}\right)$. After $30 \mathrm{~min}$ at $573 \mathrm{~K}$, the flow was changed to He until the MS signals were stable. At this point, the modulation experiment was started. A full modulation period ( $T=155 \mathrm{~s}$ ) consisted of a pulse of $1 \mathrm{vol} \% \mathrm{H}_{2} / \mathrm{He}$ followed by an equal pulse of 1 vol\% $\mathrm{O}_{2} / \mathrm{He}$ while recording 28 quick-EXAFS spectra ( $c a$. $5.3 \mathrm{~s}$ per spectrum). The full ME-EXAFS experiment consisted of thirty consecutive periods. The first two periods were discarded in the data analysis in order to ensure that steady state conditions have been achieved. The PSD data are obtained using a homemade Matlab based routine including also the averaging of the time-resolved spectra over the modulation periods.

\section{Results and discussion}

\subsection{Interpretation of the phase resolved EXAFS spectra}

The fundamental requirement in order to apply the PSD function (eqn (1)) is that the system undergoes a reversible transformation in response to the external stimulation. This is the case for many phenomena occurring at the surface or at the bulk of solid materials, such as adsorption/desorption of probe molecules or redox dynamics, which are often involved in catalytic or gas sensing mechanisms. Under this condition, a sinusoidal stimulation of frequency $\omega$ provokes a likewise sinusoidal system variation characterized by the same frequency $\omega$, but with an amplitude and eventually a phase delay $\phi$, both depending on the system response kinetics. Considering that an ideal $\mathrm{Pd}$ surface during the redox modulation experiment consisting of $\mathrm{H}_{2} / \mathrm{O}_{2}$ pulses $\mathrm{Pd}$ oscillates between two boundary structures, i.e. fully reduced and partially oxidized, with frequency $\omega$. These two structures generate EXAFS spectra $\chi_{t}(k)_{\mathrm{i}}$ and $\chi_{t}(k)_{\mathrm{f}}$, whose difference spectrum can be expressed as $\Delta \chi_{t}(k)=\chi_{t}(k)_{\mathrm{f}}-\chi_{t}(k)_{\mathrm{i}}$. These two spectra are not necessarily the first and last spectra of a stimulation period. Moreover, at each $k$ value, the temporal variation of the EXAFS signal intensity $\chi_{k}(t)$ oscillates between a maximum $\left(\chi_{k, \max }\right)$ and a minimum $\left(\chi_{k, \text { min }}\right)$ value, hence with an amplitude equal to $\Delta \chi_{k} / 2\left(\Delta \chi_{k}=\chi_{k, \max }-\chi_{k, \text { min }}\right)$.

It must be noticed that the PSD function (eqn (1)) is applied to the signal variation recorded along the time at each $k$ value $\chi_{k}(t)$, by varying the demodulation phase angle in the range $0^{\circ}<$ $\varphi^{\mathrm{PSD}}<360^{\circ}$ with a step size of $10^{\circ}$. This operation transforms $\chi_{k}(t)$, recorded in the time domain, into a new set of $36 \chi_{k}\left(\varphi^{\mathrm{PSD}}\right)$, representing the phase domain. The new phase-resolved EXAFS spectrum at a given $\varphi^{\mathrm{PSD}}$ angle, $\chi_{\varphi}(k)$ is obtained by extending this procedure to all of the $0<k<14$ values. The strength of PSD is to filter those contributions of the spectrum that are unaltered by the external stimulation, together with a considerable increase of the signal to noise ratio. ${ }^{8,9}$ As a result, a phase-resolved spectrum contains only those features of the active (responsive) species to the stimulation.

The signal intensity variation in the phase domain, $\chi_{k}\left(\varphi^{\mathrm{PSD}}\right)$, can be calculated from eqn $(1):^{3}$

$$
\chi_{k}\left(\varphi^{\mathrm{PSD}}\right)=\frac{\Delta \chi_{k}}{2} \cos \left(\phi-\varphi^{\mathrm{PSD}}\right)
$$

where $\phi$ is the phase delay with respect to the stimulation (phase lag). It follows from eqn (3) that while the variation of $\chi_{k}$ follows a sine function in the time domain, it exhibits a cosine function in the phase domain. Hence, the amplitude of the phase-resolved EXAFS spectra $\chi_{\varphi}(k)$ has a maximum when $\cos \left(\phi-\varphi^{\mathrm{PSD}}\right)=1$, i.e. for $\varphi^{\mathrm{PSD}}=\phi$, the so called "in-phase" spectrum; it is zero when $\cos \left(\phi-\varphi^{\mathrm{PSD}}\right)=0$, i.e. for $\varphi^{\mathrm{PSD}}=\phi+$ $90^{\circ}$; and it has a minimum when $\cos \left(\phi-\varphi^{\mathrm{PSD}}\right)=-1$, i.e. for $\varphi^{\mathrm{PSD}}=\phi+180^{\circ}$. This trend generates the typical envelop of the phase-resolved spectra. The amplitude of the in-phase spectrum corresponds to half of the overall signal variation within one modulation period, $\Delta \chi_{k} / 2$. In other words, a difference spectrum $\Delta \chi(k)$ will exhibit twofold amplitude compared to that of the phase-resolved spectrum. In practice the amplitude of the phase-resolved spectrum also depends on the method adopted to calculate the integral of eqn (1). The Simpson series has been used here and in the previous studies, which slightly underestimates the integral. ${ }^{6-8,12}$ Thus, an amplitude correction factor, $\alpha$, can be introduced. In this work, we have graphically evaluated the correction factor by dividing a simulated difference spectrum by the in-phase spectrum (Fig. 1A), and we found $\alpha=1.05$. Hence, the following relation can be derived from eqn (3) extended to the whole $\chi(k)$ spectrum:

$$
\frac{2 \alpha}{\cos \left(\phi-\varphi^{\mathrm{PSD}}\right)} \chi_{\varphi^{\mathrm{PSD}}}(k)=\Delta \chi(k) \approx \chi_{t}(k)_{f}-\chi_{t}(k)_{i}
$$




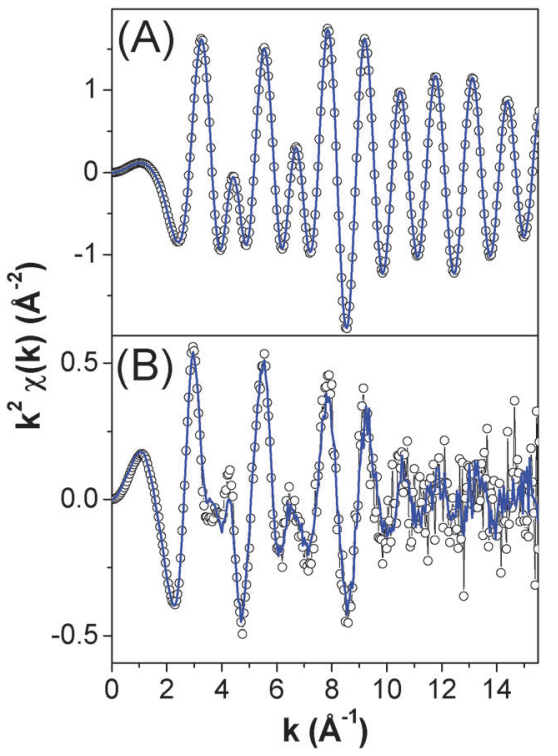

Fig. 1 Comparison of (A) simulated and (B) experimental (1.6 wt\% $\left.\mathrm{Pd} / \mathrm{Al}_{2} \mathrm{O}_{3}\right) k^{2}$-weighted EXAFS spectra at the Pd K-edge during a modulation experiment. Open symbols: difference time-resolved spectrum between the two extreme states, $\chi(k)_{f}-\chi(k)_{i}$. Solid line: "in-phase" spectrum (intensity multiplied by 2.1). The deviation of the solid line from the difference spectrum in (B) is due to the noise attenuation property of PSD. Experimental data were obtained at $573 \mathrm{~K}$ with 1 vol\% $\mathrm{H}_{2} / \mathrm{Ar}$ and 1 vol\% $\mathrm{O}_{2} / \mathrm{Ar}$ on $1.6 \mathrm{wt} \% \mathrm{Pd} / \mathrm{Al}_{2} \mathrm{O}_{3}$.

Eqn (4) represents the fundamental mathematical relation between the time- and the phase-resolved spectra. In particular, once the experimental spectrum of the initial state $\chi_{t}(k)_{\mathrm{i}}$ is known it is possible to "extrapolate" from the phase-resolved $\chi_{\varphi}(k)$ spectrum that of a final or even of intermediate state $\chi_{t}(k)_{\mathrm{f}} \cdot{ }^{16}$ This will be further shown in Section 3.2. The $\approx$ symbol in eqn (4) highlights the essential advantage of the phaseresolved spectrum with respect to the experimental difference spectrum, i.e. the increase of the signal-to-noise ratio. ${ }^{8,9}$ This advantage is demonstrated in Fig. 1. Indeed, in the case of simulated noise-free EXAFS spectra at the Pd K-edge the in-phase spectrum (Fig. 1A, solid line) scaled by 2.1 , i.e. by $2 \alpha$ according to eqn (4), perfectly matches the difference spectrum (empty circles), therefore confirming the validity of eqn (4). A similar result was obtained with the experimental spectra recorded during a $\mathrm{H}_{2}$ vs. $\mathrm{O}_{2}$ redox modulation at $573 \mathrm{~K}$ over $1.6 \mathrm{wt} \% \mathrm{Pd} / \mathrm{Al}_{2} \mathrm{O}_{3}$ (Fig. 1B). In this case, the scaled in-phase spectrum (solid line), exhibits less noise compared to the difference spectrum obtained from the spectra of the most oxidized and the most reduced states of the supported Pd nano-particles (empty circles).

Because $\chi_{t}(k)$ are oscillating functions, there are points along the $k$-axis where $\chi_{t}(k)_{\mathrm{i}}$ and $\chi_{t}(k)_{\mathrm{f}}$ cross each other (Fig. 2A). In these points, $\Delta \chi_{k}=0$ and consequently $\chi_{k}\left(\varphi^{\mathrm{PSD}}\right)=0$ for any $\varphi^{\mathrm{PSD}}$ (eqn (3)). These points are nodes of the phase resolved EXAFS spectra. The plots of all of the phase-resolved EXAFS spectra obtained in the phase domain $0^{\circ}<\varphi^{\mathrm{PSD}}<360^{\circ}$ result in a linear front (amplitude at each $k$ value given by eqn (3)) of waves propagating along the $k$-axis, all crossing the $k$-axis at the nodes, giving rise to a typical envelope as the one displayed in the bottom panel of Fig. 2A. As shown in Fig. 3, the envelop can exhibit two characteristic shapes, "in phase" (Fig. 3A) or "out of phase" (Fig. 3B) (not to be confused with the "in-phase" spectrum), depending on the variation of the possible $3 n$ EXAFS variables. Contrary to the "in phase", the "out of phase" shape is generated when at least one of the possible $3 n$ variables exhibits a different phase lag $\phi_{i}$ with respect to the others. A different phase delay can occur either between the structural parameters of a single shell $\left(N_{i}, R_{i}\right.$ and $\left.\sigma_{i}^{2}\right)$ or of different shells. These two scenarios can be distinguished by means of the
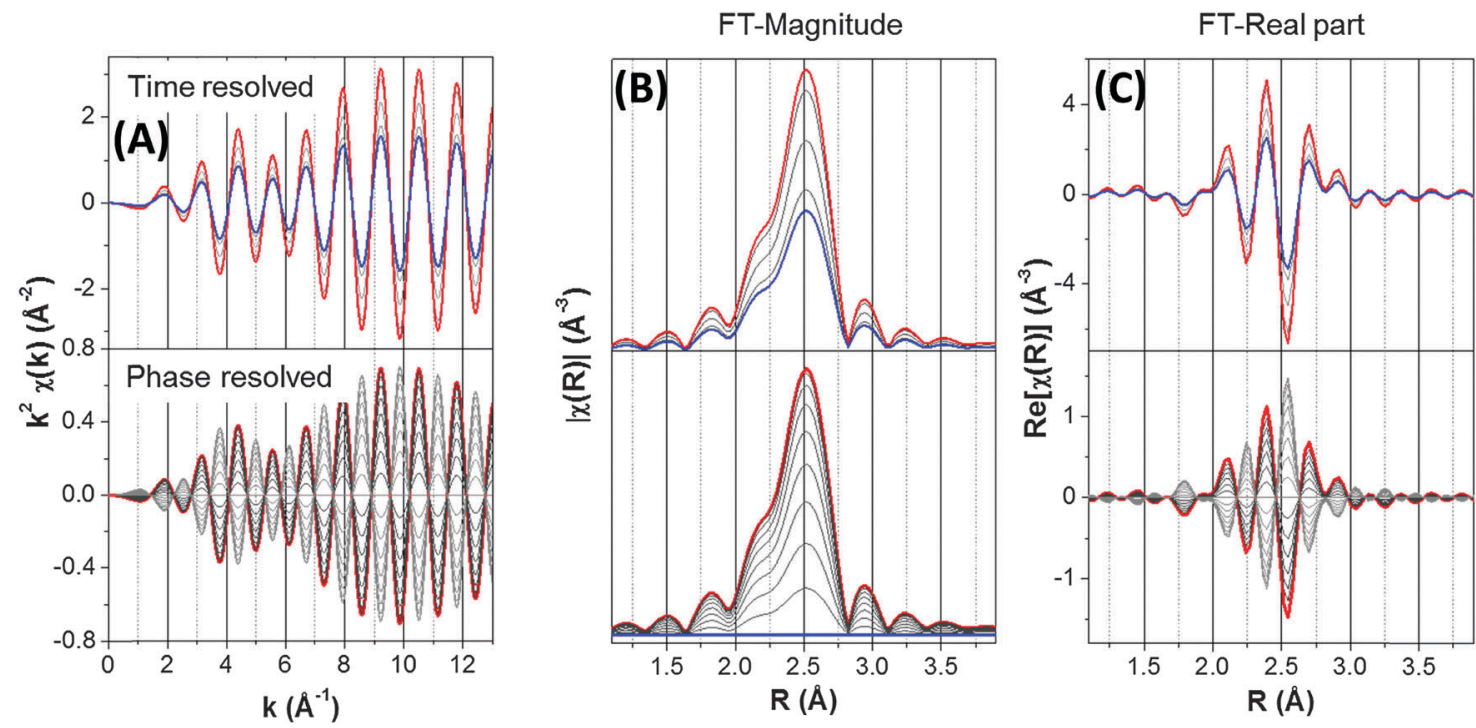

Fig. 2 Effect of variation of coordination number $N$ (according to Fig. S2, ESI $\dagger$ ) of a single Pd-Pd first coordination shell on (A) the simulated $k^{2}$-weighted $\chi(k)$ EXAFS spectra, (B) the FT-magnitude $|\chi(R)|$ and $(C)$ the FT-real part Re $[\chi(R)]$. Top panels: simulated time-resolved spectra where $N$ varies from 12 (red curve) to 6 (blue curve) with a phase lag $\phi=0^{\circ}\left(R=2.758 \AA, \sigma^{2}=0.003 \AA^{2}, \Delta E^{\circ}=0 \mathrm{eV}\right)$. Bottom panels: corresponding phase-resolved spectra, where the red curve is the "in-phase angle" ( $\left.\varphi^{\mathrm{PSD}}=\phi=0^{\circ}\right)$. 


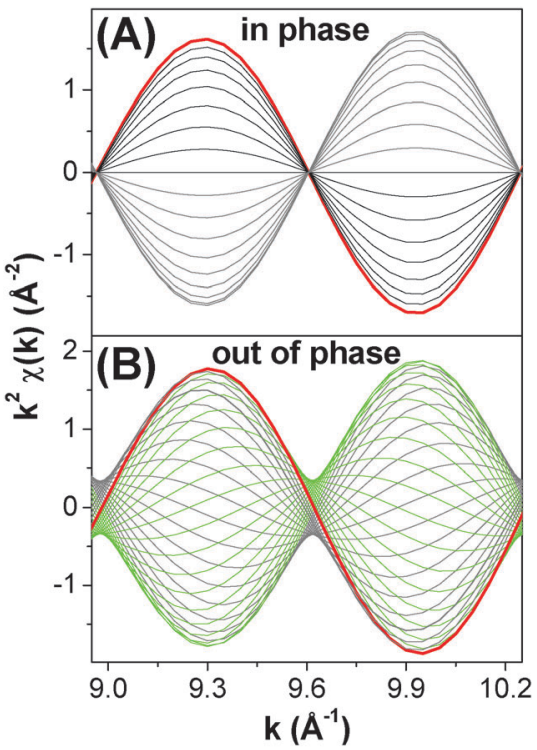

Fig. 3 Effect of phase lag $\phi$ on the phase-resolved $k^{2} \chi(k)$ EXAFS spectra of simulated $\mathrm{Pd}-\mathrm{Pd}$ first coordination shell with variation of both coordination number $(6<N<12)$ and interatomic distance $(2.7577 \AA<R<$ $2.8577 \AA$ ) $) \cdot \sigma^{2}=0.003 \AA^{2} ; \Delta E^{\circ}=0 \mathrm{eV}$. (A) $N$ and $R$ have same phase lag $\left(\phi_{N}=\right.$ $\phi_{R}=0^{\circ}$ ) and generate an "in phase" envelop; (B) $N$ and $R$ have different phase lag $\left(\phi_{\mathrm{N}}=0^{\circ}\right.$ and $\left.\phi_{\mathrm{R}}=-40^{\circ}=320^{\circ}\right)$ and generate an "out of phase" envelop (red curve $\varphi^{\mathrm{PSD}}=\phi_{\mathrm{R}}=-40^{\circ}$; gray curves: $-40^{\circ}<\varphi^{\mathrm{PSD}}<-40^{\circ}+$ $180^{\circ}$; green curves $-40^{\circ}+180^{\circ}<\varphi^{\mathrm{PSD}}<-40^{\circ}+360^{\circ}$.

analysis of the Fourier transform of the phase-resolved EXAFS spectra, $\chi_{\varphi}(R)$. The FT-EXAFS spectra are calculated according to the equation:

$$
\chi(R)=\int_{k_{i}}^{k_{f}} W(k) k^{n} \chi(k)[\cos (2 \pi k R)+i \sin (2 \pi k R)] \mathrm{d} k
$$

where $W(k)$ is a window function which dampens the extremes of $\chi(k)$ (the Hanning window function with $\mathrm{d} k=1$ was used) and $k^{n}$ is the $k$-weighting. Eqn (5) shows that $\chi(R)$ contains both real $(\operatorname{Re}[\chi(R)])$ and imaginary $(\operatorname{Im}[\chi(R)])$ parts, which are combined to calculate the magnitude of $\chi(R)$ affording an envelope of the real and imaginary parts:

$$
|\chi(R)|=\sqrt{(\operatorname{Re}[\chi(R)])^{2}+(\operatorname{Im}[\chi(R)])^{2}}
$$

It is important to note that $|\chi(R)|$ always exhibits positive values, whereas its real and imaginary parts oscillate between positive and negative values. Moreover, $\operatorname{Re}\left[\chi_{\varphi=\phi}(R)\right]=-\operatorname{Re}\left[\chi_{\varphi=\phi+180^{\circ}}(R)\right]$, because the amplitude of the real and imaginary parts of the phase-resolved FT spectra as a function of $\varphi^{\mathrm{PSD}}$ are proportional to $\cos \left(\phi-\varphi^{\mathrm{PSD}}\right)$ (eqn (3)), whereas $\left|\chi_{\varphi=\phi(R) \mid}=\right| \chi_{\varphi=\phi+180^{\circ}}(R) \mid$. In other words, the magnitude of the FT-EXAFS spectra in the range $\phi<\varphi^{\mathrm{PSD}}<$ $\phi+90^{\circ}$ overlaps with those in the range $\phi+90^{\circ}<\varphi^{\mathrm{PSD}}<180^{\circ}+\phi$ (Fig. 2B), whereas the real and the imaginary parts of the latter set of spectra are the reciprocals of the former set (Fig. 2C). This observation is crucial to solve the dynamics of one coordination shell relative to another one, e.g. one shell appears while the other one disappears during a structural transformation, as will be described in the following sections.

\subsection{Simulated EXAFS spectra at the Pd K-edge}

The simple ME-EXAFS experiment considered here consists of alternate and repeated switches from $\mathrm{H}_{2}$ (i.e. reducing atmosphere) to $\mathrm{O}_{2}$ (i.e. oxidizing atmosphere) over an extended metallic Pd surface. This surface simulates for simplicity a metal nano-particle exposing extended facets as it is in the case of the nano-structured Pd supported catalyst (1.6 wt\% $\mathrm{Pd} / \mathrm{Al}_{2} \mathrm{O}_{3}$ ). Under such conditions, Pd periodically (either partially or fully) reduces and re-oxidizes. Depending on its extent, the oxidation process may cause a decrease of the average coordination number of the first Pd-Pd shell. The metal Pd nano-particle becomes increasingly smaller because the oxidation starts from the surface. Simultaneously, the oxidation leads to the formation of the two main $\mathrm{PdO}$ coordination shells, i.e. the first $\mathrm{Pd}-\mathrm{O}$ and the second Pd-Pd (hereafter labeled as Pd-(O)-Pd) coordination shells. The decrease of the particle size can eventually produce a small decrease of the interatomic distance $R$ because of lattice contraction and also an increase of the disorder $\left(\sigma^{2}\right)$. In a first approximation, the variation of these parameters $\left(N_{i}, R_{i}\right.$ and $\left.\sigma_{i}^{2}\right)$ within the time $I(t)$, resulting from a sinusoidal stimulation of frequency $\omega$, can be represented using a sine function oscillating between a maximum $\left(I_{\max }\right)$ and a minimum $\left(I_{\min }\right)$ value with an amplitude equal to $\Delta I / 2\left(\Delta I=I_{\max }-I_{\min }\right)$ :

$$
I(t)=\left(I_{\min }+\frac{\Delta I}{2}\right) \pm \frac{\Delta I}{2} \sin \left(\omega t+\phi_{i}\right)
$$

where $\phi_{i}$ is the relative phase lag of each parameter response with respect to the external stimulation and the + or - sign depends on whether the considered parameter increases or decreases along a stimulation period, respectively. Because - sin $\alpha=\sin \left(\alpha+180^{\circ}\right)$, the two parameters propagating as two antiphase sine functions possess the same relative phase lag $\phi_{i}$, but one of them has an absolute phase lag of $\phi_{i}+180^{\circ}$. Moreover, the parameters can either possess the same or a different relative phase lag, $\phi_{i}$. Both possibilities have been considered for the simulation. It should be pointed out that in the ME approach it is not crucial that the system response follows a perfect sine function. However, it is necessary that it is stationary along the time (i.e. it must be fully reversible) and a given event has to occur at the same frequency as that of the stimulation.

3.2.1 Effect of the variation of $N, \sigma^{2}$, and $R$ of a single shell. The effect of the variation of $N, R$ and $\sigma^{2}$ on the $k$ space EXAFS spectrum $\chi(k)$ can be easily understood from eqn (1).

(1) $N$ is directly proportional to the amplitude of $\chi(k)$. Fig. 2 shows the comparison of the simulated noise-free timeresolved $\chi(k),|\chi(R)|$ and $\operatorname{Re}[\chi(R)]$ of a single Pd-Pd shell (upper panels) with the corresponding phase-resolved spectra (lower panels), for a sinusoidal variation of only $N$ (Fig. S1, ESI $\dagger$ ) between 12 (red line in Fig. 2A) and 6 (blue line in Fig. 2A). In this case, the in-phase phase-resolved spectrum (red line in Fig. 2A) is simply equal to the time-resolved spectrum obtained with $N_{\max }=12$ (same frequency and disorder, because $R$ and $\sigma^{2}$ have been kept constant) but with an amplitude reduced by a factor of 4.2 (i.e. $2.1 N_{\max } / \Delta N$, with $\Delta N=6$ ). Thus, the fitting of the in-phase FT spectrum $\left|\chi_{\varphi=\phi}(R)\right|$ gives $R$ and $\sigma^{2}$ of the time-resolved 
spectra and $N=\Delta N / 2.1$. Moreover, as shown in Fig. 4, the normalized (i.e. divided by the maximum value) time-resolved real part $\operatorname{Re}[\chi(R)]$ is equal to the normalized phase-resolved $\operatorname{Re}\left[\chi_{\varphi}(R)\right]$.

(2) $\sigma^{2}$ affects the amplitude as well (Fig. S2 and S3, ESI $\dagger$ ). However, the variation is $k$-dependent because $\sigma^{2}$ appears in the exponential term $\exp \left(-2 \sigma^{2} k^{2}\right)$ of eqn (1). As a consequence the amplitude of $\chi(k)$ decreases along the $k$-axis with increasing $\sigma^{2}$. In particular, $\sigma^{2}$ affects the shape of the FT-EXAFS spectrum. The variation of $\sigma^{2}$ can be easily distinguished by the variation of $N$ because it leads to a distortion of the real part with respect to that of the initial state. This effect can be better observed by comparing the normalized in-phase $\operatorname{Re}[\chi(k)]$ (e.g. divided by the maximum value) with that of the initial state (Fig. S4, ESI $\dagger$ ).

(3) In marked contrast, the change of interatomic distance $R$ reflects upon both the amplitude and the frequency of the FT functions (Fig. S5 and S6, ESI $\dagger$ ). As a consequence, the phaseresolved EXAFS spectrum is a difference of two waves (eqn (4)) having frequency $R$ and $R+\Delta R$. However, $\Delta R$ is usually lower than the achieved spatial resolution in EXAFS spectroscopy, because of the $|\chi(R)|$ peak broadening due to the limited $k$ range (in this case $2<k<14 \AA^{-1}$ ). Hence, the two peaks overlap in the phase-resolved FT spectra and the signals with frequency $R$ and $R+\Delta R$ are additive in the real and imaginary parts of the FT. This latter property makes the normalized real part of the phase-resolved spectra slightly shifted along $R$ and with lower (higher) amplitude at lower (higher) $R$ compared to the normalized part of the initial state (Fig. 4).

The variation of $R$ plays a dominant role in deciding the shape of the phase-resolved spectra. The differentiation of the synchronous variation of $N$ is difficult if the two parameters possess the same relative phase lag, $\phi_{\mathrm{N}}=\phi_{\mathrm{R}}$ (Fig. S7 and S8, ESI $\dagger)$. In contrast, if the two parameters have different phase lag (Fig. S9, ESI $\dagger$ ), the phase-resolved $\chi(k)$ spectra generate an out of phase envelop (Fig. 3 and 5) and the final state with bond distance $R+\Delta R$ can be isolated in the FT spectra at $\varphi^{\mathrm{PSD}}=\phi_{\mathrm{N}}+90^{\circ}$ (green curves in Fig. 5). The out of phase

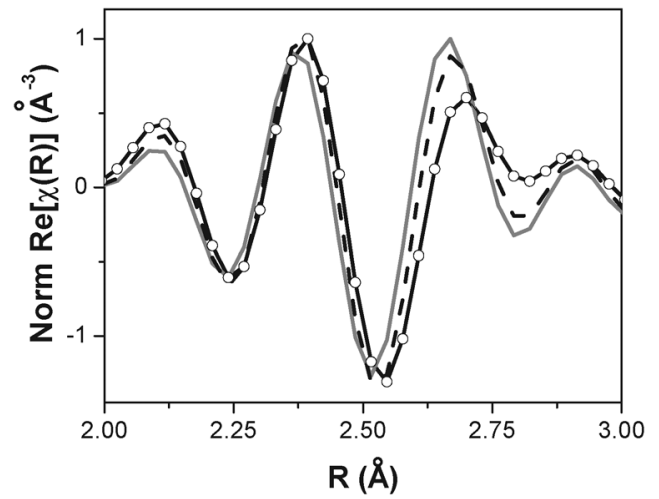

Fig. 4 Comparison of simulated normalized $k^{2}$-weighted $\operatorname{Re}[\chi(R)]$ of a $\mathrm{Pd}-\mathrm{Pd}$ first coordination shell $\left(N=12, R=2.7577 \AA, \sigma^{2}=0.003 \AA^{2}, \Delta E^{\circ}=\right.$ $0 \mathrm{eV}$; empty circles) with those of the "in-phase angle" $\operatorname{Re}\left[\chi_{\varphi=\phi}(R)\right]$ upon variation of only $N(6<N<12$; black curve), only $R(2.7577 \AA<R<$ $2.8577 \AA$; grey curve) and both $N$ and $R$ with the same phase lag (dash curve). envelop can be observed again in the real part of the phaseresolved FT spectra.

3.2.2 Effect of the variation of different coordination shells. In the course of an oxidation process the $\mathrm{Pd}-\mathrm{Pd}$ shell gradually loses intensity while the $\mathrm{Pd}-\mathrm{O}$ and the $\mathrm{Pd}-(\mathrm{O})-\mathrm{Pd}$ shells intensify with a consequent increase or decrease of the corresponding coordination numbers. As a result, the timeresolved signals of these two shells are in anti-phase with respect to that of the Pd-Pd shell. Furthermore, if the oxidation starts at the surface and proceeds towards the bulk, the formation of the $\mathrm{Pd}-(\mathrm{O})-\mathrm{Pd}$ shell might occur with a different phase lag compared to that of the $\mathrm{Pd}-\mathrm{O}$ shell. On the other hand, if the reduction process is faster than the re-oxidation, the variation of the $\mathrm{Pd}-\mathrm{O}$ and $\mathrm{Pd}-(\mathrm{O})-\mathrm{Pd}$ shells might occur with a larger phase lag than the one of the Pd-Pd shell. As demonstrated in Section 3.1.1, a parameter with a different phase lag with respect to the others generates a set of out of phase phase-resolved EXAFS spectra. Fig. 6B and C show the situation in which all shells vary synchronously, whereas Fig. 6D the situation in which one shell presents a phase lag with respect to the others. If one considers the isolated shells in the reference spectra of Pd metal foil and bulk PdO (Fig. 6A), in principle the three different shells can be solved by means of the Fourier transformation of the phase-resolved spectra (Fig. 6B-D). In particular, the Pd-O shell appears at ca. $1.5 \AA$ and is far enough from the Pd-Pd shell (ca. 2.5 $\AA$ ) to allow for their separation in the FT spectra. In contrast, the Pd-(O)-Pd shell partially overlaps with the Pd-Pd shell (Fig. 6A). Hence, in a real system, where reduced and oxidized states coexist, the separation of the contribution of one of the two shells may be difficult if it represents only a minute fraction of the whole system. With the assumption that the exposure time to the $\mathrm{O}_{2}$-rich atmosphere is short enough, our simulation considers only partial oxidation of the Pd surface, i.e. the formation of a single layer of PdO. Therefore, the average coordination number $N$ of the $\mathrm{Pd}-(\mathrm{O})-\mathrm{Pd}$ shell has to be rather small (this is confirmed by our fitting in Table 1; vide infra). As a result, in the case that all three shells possess the same phase lag (Fig. 6C), the $\mathrm{Pd}-(\mathrm{O})-\mathrm{Pd}$ shell appears in the phase-resolved FT spectra as a shoulder on the right side of the Pd-Pd shell. The difference in the case where no $\mathrm{Pd}-(\mathrm{O})-\mathrm{Pd}$ shell is taken into consideration is rather obvious (Fig. 6B). A more realistic situation occurs when reduction is faster than the re-oxidation, ${ }^{8}$ and the $\mathrm{Pd}-(\mathrm{O})-\mathrm{Pd}$ shell varies with a different phase lag compared to that of Pd-Pd (Fig. 6D). In this case, PSD has the advantage to clearly isolate the contribution of this shell at a specific phase-angle (thick black curve in the middle panel of Fig. 6D; ca. 2.75 ̊). This situation is similar to that described above when the interatomic distance $R$ of a single shell varies with a different phase lag. These two similar situations can be distinguished from each other upon inspection of the real part of the phase-resolved FT spectra. The variation of $R$ affects the whole real part of the phase-resolved FT spectra of that specific shell as shown in Fig. 7A for the Pd-Pd shell. In contrast, the variation of a second shell with a given phase lag perturbs only a specific section of the phase-resolved spectra, where the 

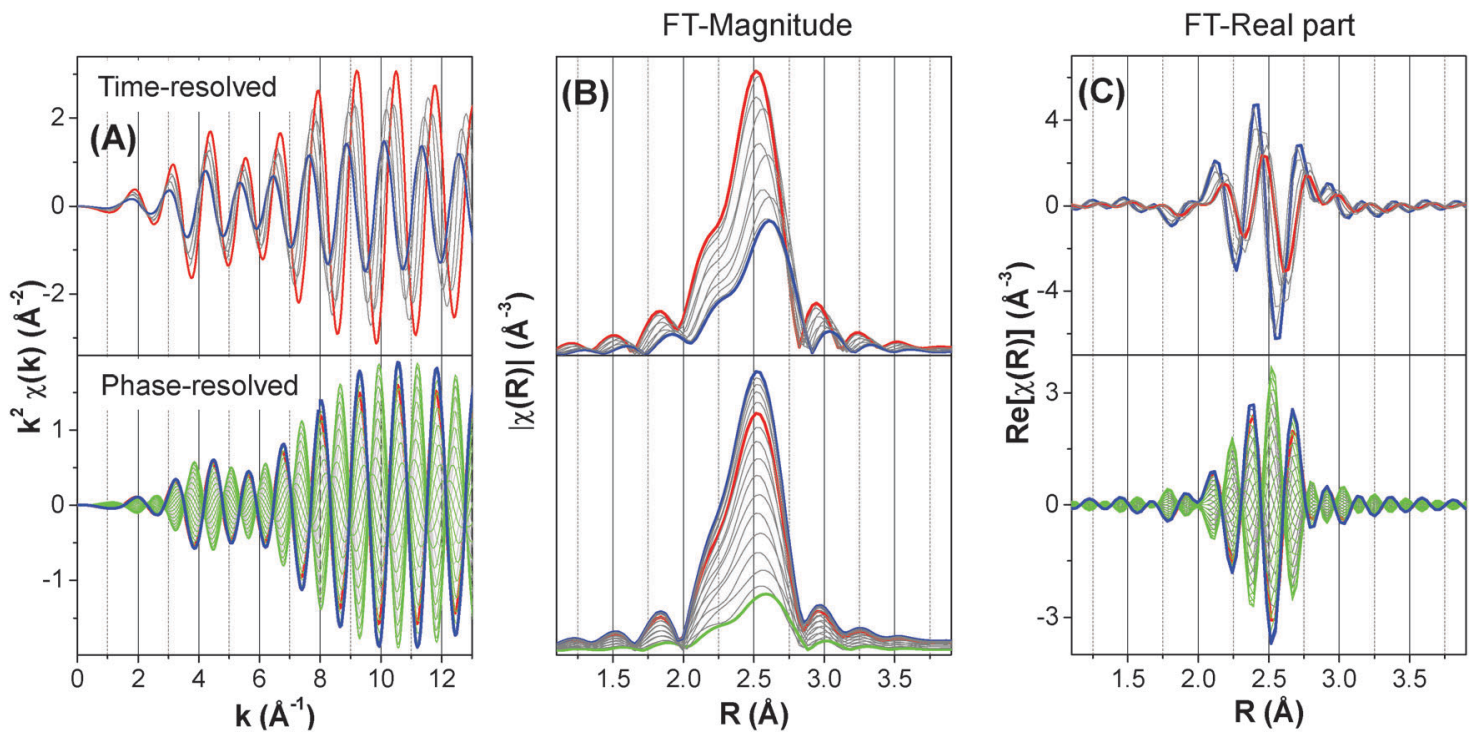

Fig. 5 Effect of simultaneous variation of the coordination number $N$ and interatomic distance $R$ of a single Pd-Pd first coordination shell with different phase lag on (A) the simulated $k^{2}$-weighted EXAFS spectra; (B) the FT-magnitude, $|\chi(R)|$; and (C) the FT-real part, Re[ $\left.\chi(R)\right]$. Top panels: simulated timeresolved spectra where $N$ and $R$ vary from 12 and $2.7577 \AA$ (red) to 6 and $2.8577 \AA$ (blue) with a phase lag $\phi_{\mathrm{N}}=0^{\circ}$ and $\phi_{\mathrm{R}}=320^{\circ}\left(\sigma^{2}=0.003 \AA^{2}, \Delta E^{\circ}=\right.$ $0 \mathrm{eV}$ ). Bottom panels: corresponding phase-resolved spectra, where the red curve corresponds to $\varphi^{\mathrm{PSD}}=\phi_{\mathrm{N}}=0^{\circ}$, the blue curve to the "in-phase angle" $\left(\varphi^{\mathrm{PSD}}=\phi_{\mathrm{R}}=320^{\circ}\right)$ and the green curves to $\phi_{\mathrm{R}}+180^{\circ}<\varphi^{\mathrm{PSD}}<\phi_{\mathrm{R}}$

contributions of the real parts of the two shells overlap as has been demonstrated for the Pd-Pd and Pd-(O)-Pd shells (the 2.6-2.9 $\AA$ region, Fig. 7B). This feature of PSD is crucial for the structural analysis of phase-resolved data and has been exploited to isolate the spectral features of a third component beside the two boundary states between which the system has been oscillating during the modulation experiment. ${ }^{16,22}$

One more piece of information can be obtained from the analysis of the magnitude and the real part of the phase-resolved FT spectra. PSD provides access to the relative dynamics of the different shells, i.e. whether they appear simultaneously or delayed with respect to each other, or one appears while another one disappears. In case the evolution of shells is delayed, the considered shells are in 'anti-phase' and this is reflected in the variation of the real part as a function of the phase angle. This concept is clarified in Fig. 8A, where the phase angular variation of the characteristic interatomic distance $R$ (obtained from the FT-spectra) for the three Pd-O, Pd-Pd and Pd-(O)-Pd shells is plotted. As mentioned above, because the magnitude of FT possesses only positive values the negative values can be obtained by considering the real (or alternatively the imaginary) part (Fig. 8B). In the case of the real part of FT, it is important to plot the intensity of a characteristic $R$, where that specific shell exhibits the maximum amplitude. For example, for the Pd-Pd shell $R$ is chosen at $2.512 \AA$. However, if two shells are close to each other and they partially overlap, as it is in the case of Pd-Pd and $\mathrm{Pd}-(\mathrm{O})-\mathrm{Pd}$, it is important to select a characteristic $R$, where the two shells do not overlap. Following our example, $R$ is $2.393 \AA$ and $2.976 \AA$ for Pd-Pd and Pd-(O)-Pd, respectively in Fig. 8B rather than 2.512 and $2.792 \AA$, respectively. For this purpose, it might also be useful to compare the imaginary part of the phaseresolved FT spectra. Fig. 8B shows that the $\mathrm{Pd}-\mathrm{O}$ and $\mathrm{Pd}-\mathrm{Pd}$ shells are in anti-phase, whereas the Pd-(O)-Pd shell is out-ofphase relative to the Pd-O shell and $\phi_{\mathrm{r}}=-40^{\circ}$. The minus sign indicates that this shell appears with a time delay of $t_{\mathrm{d}}$ with respect to the Pd-O shell, in this case $t_{\mathrm{d}}=\phi_{\mathrm{r}} T / 360^{\circ}=-40^{\circ} \times 100$ $\mathrm{s} / 360^{\circ}=-11.1 \mathrm{~s}$. Therefore, the Pd-(O)-Pd shell starts to appear $11.1 \mathrm{~s}$ after the $\mathrm{Pd}-\mathrm{O}$ shell, in agreement with the simulation.

\subsection{Experimental ME-EXAFS spectra at the Pd K-edge}

In order to demonstrate our approach, $1.6 \mathrm{wt} \% \mathrm{Pd} / \mathrm{Al}_{2} \mathrm{O}_{3}$ was subjected to 30 consecutive $1 \mathrm{vol} \% \mathrm{O}_{2} / \mathrm{He}$ to $1 \mathrm{vol} \% \mathrm{H}_{2} / \mathrm{He}$ switches at $573 \mathrm{~K}$ with a modulation period of $155 \mathrm{~s}$.

The first time-resolved spectra recorded at the beginning of the modulation experiment reveals that the Pd is oxidized (no contribution of the metallic Pd-Pd shell can be distinguished in the FT-EXAFS spectrum), as expected after the thermal pretreatment at $573 \mathrm{~K}$ in 1 vol\% $\mathrm{O}_{2} / \mathrm{He}$ (Fig. S11, ESI $\dagger$ ). However, Pd readily reduces already after the first exposure to $1 \mathrm{vol} \%$ $\mathrm{H}_{2} / \mathrm{He}$ and steady state conditions were reached after the first two cycles. Thus, the time-resolved spectra recorded during the first two cycles were removed from the subsequent data processing.

The averaged time-resolved XANES spectra are shown in Fig. 9. A variation of the whiteline region upon oxidation is clearly visible. The linear combination analysis of the experimental spectra using those of bulk PdO and Pd foil as the reference spectra (inset of Fig. 9) shows that Pd undergoes a fast reduction in hydrogen, whereas the re-oxidation is slower and only $40 \%$ of the $\operatorname{Pd}(0)$ is oxidized to $\operatorname{Pd}(\mathrm{II})$ at the end of the oxygen pulse. The corresponding time-resolved FT-EXAFS spectra (Fig. 10A) recorded in hydrogen are dominated by a single contribution centered at $2.485 \AA$ corresponding to the first Pd-Pd coordination shell of metallic Pd. The amplitude of the shell decreases, but does not disappear, after switching to $\mathrm{O}_{2}$. 

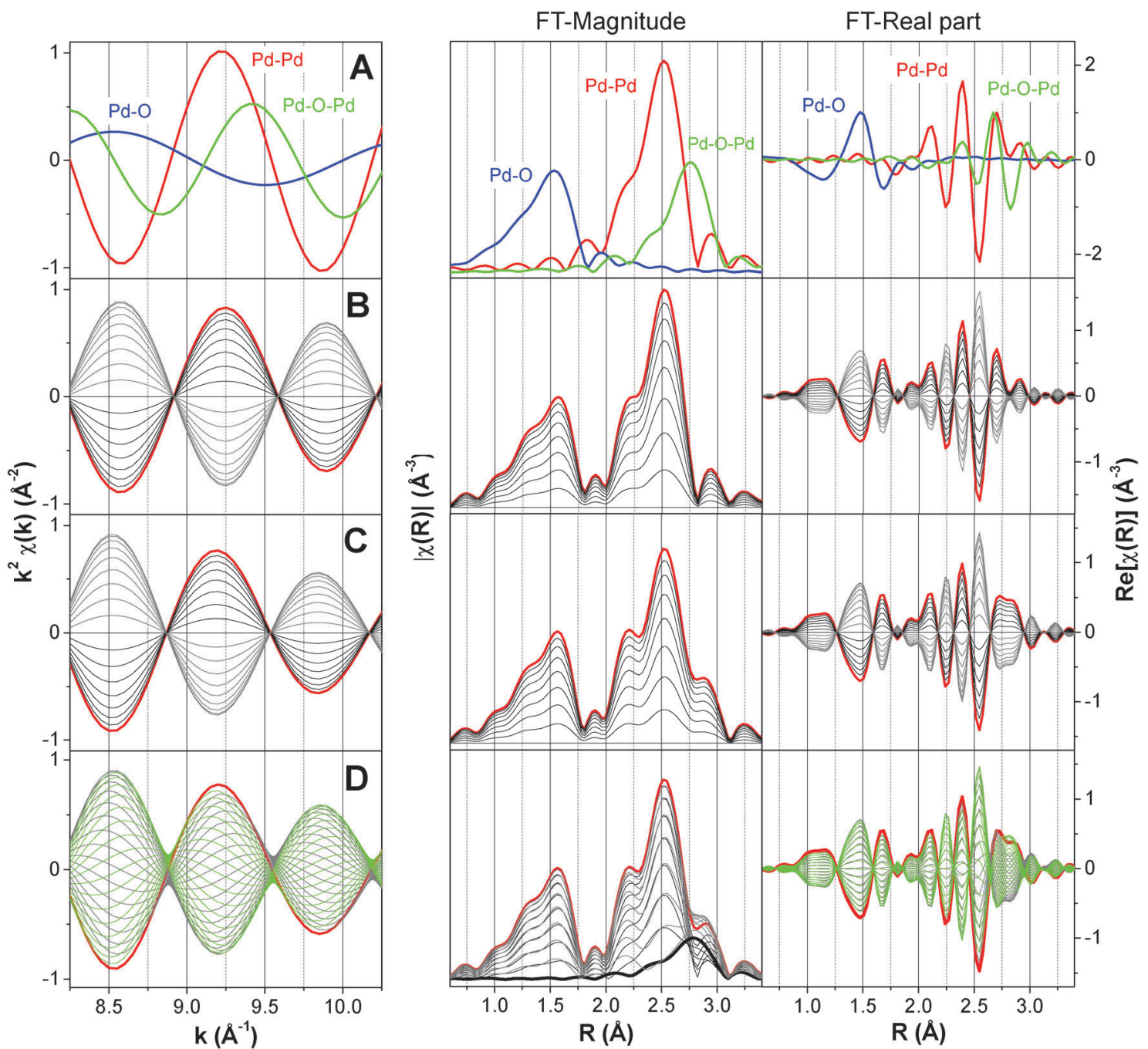

Fig. 6 Effect on the simulated phase-resolved EXAFS spectra at the Pd K-edge $\left(0^{\circ}<\varphi^{\mathrm{PSD}}<360^{\circ}\right)$ of the simultaneous variation of $(\mathrm{B})$ both $\mathrm{Pd}-\mathrm{O}$ and $\mathrm{Pd}-\mathrm{Pd}$ shells with the same phase lag, $(\mathrm{C}) \mathrm{Pd}-\mathrm{O}, \mathrm{Pd}-\mathrm{Pd}$ and $\mathrm{Pd}-(\mathrm{O})-\mathrm{Pd}$ shells with the same phase lag and (D) $\mathrm{Pd}-\mathrm{O}$ and $\mathrm{Pd}-\mathrm{Pd}$ shells with the same phase lag and $\mathrm{Pd}-(\mathrm{O})-\mathrm{Pd}$ with different phase lag. (A) Theoretical $k^{2}$-weighted $\chi(k)$ spectra of the $\mathrm{Pd}-\mathrm{Pd}, \mathrm{Pd}-\mathrm{O}$ and $\mathrm{Pd}-(\mathrm{O})-\mathrm{Pd}$ shells. Left panels: $k^{2}$-weighted $\chi(k)$. Middle panels: FT-magnitude, $|\chi(R)|$. Right panels: FT real part, $\operatorname{Re}[\chi(R)]$.

Table 1 Fit results of the $k^{3} \chi(k)$ spectra at the Pd K-edge of $1.6 \mathrm{wt} \% \mathrm{Pd} / \mathrm{Al}_{2} \mathrm{O}_{3}$ obtained in the 1 vol\% $\mathrm{H}_{2} / \mathrm{He}$ and 1 vol\% $\mathrm{O}_{2} / \mathrm{He}$ modulation experiment at $573 \mathrm{~K}$

\begin{tabular}{|c|c|c|c|c|c|c|}
\hline Gas atmosphere & Shell & $S_{0}{ }^{2 a}$ & $N^{b}$ & $R^{c}(\AA)$ & $\sigma^{2 d}\left(\AA^{2}\right)$ & $\Delta E_{0}^{e}(\mathrm{eV})$ \\
\hline \multirow{3}{*}{$1 \mathrm{vol} \% \mathrm{O}_{2} / \mathrm{He}$} & $\mathrm{Pd}-\mathrm{Pd}^{g}$ & 0.74 & $7.7 \pm 0.8$ & $2.790 \pm 0.008$ & $0.013 \pm 0.001$ & $1.72 \pm 0.7$ \\
\hline & $\mathrm{Pd}-\mathrm{O}^{h}$ & 0.70 & $2.0 \pm 0.2$ & $2.032 \pm 0.007$ & $0.003 \pm 0.001$ & $6.3 \pm 0.9$ \\
\hline & $\mathrm{Pd}-(\mathrm{O})-\mathrm{Pd} 2^{h}$ & 0.60 & $3.4 \pm 1.3$ & $3.408 \pm 0.011$ & $0.009 \pm 0.003$ & $6.3 \pm 0.9$ \\
\hline
\end{tabular}

${ }^{a}$ Amplitude reduction factor (calculated by fitting of standard Pd foil and PdO). ${ }^{b}$ Coordination number. ${ }^{c}$ Interatomic distance. ${ }^{d}$ Debye-Waller factor (mean square disorder). ${ }^{e}$ Energy shift. ${ }^{f}$ Fit of last time-resolved spectrum recorded in $\mathrm{H}_{2}$ and representing the initial state $\left.\chi(k)\right)_{\mathrm{i}} \cdot{ }^{g}$ Fit of last time-resolved spectrum in $\mathrm{H}_{2}$ subtracted by the scaled phase-resolved spectrum at $\varphi^{\mathrm{PSD}}=230^{\circ} .{ }^{h}$ Fit of scaled phase-resolved spectrum at $\varphi^{\mathrm{PSD}}=$ $250^{\circ} .{ }^{g}$ and ${ }^{h}$ Represent the structure of the final state in $\mathrm{O}_{2}$.

Simultaneously, a second contribution appears at $1.564 \AA$ due to the formation of the first Pd-O shell, thus confirming the partial re-oxidation of Pd. The FT-EXAFS spectrum of bulk PdO (Fig. 10A) is characterized by the presence of a second broad signal due to the second Pd-(O)-Pd shell, which is composed of four Pd atoms located at $3.03 \AA$ (Pd-(O)-Pd1 shell) and eight Pd atoms located at $3.42 \AA$ (Pd-(O)-Pd2 shell). These two shells are so close to each other that they partially overlap, generating the broad peak of the FT-EXAFS spectrum centered at $3.1 \AA$ with a shoulder at lower $R$. However, the contribution of this latter 


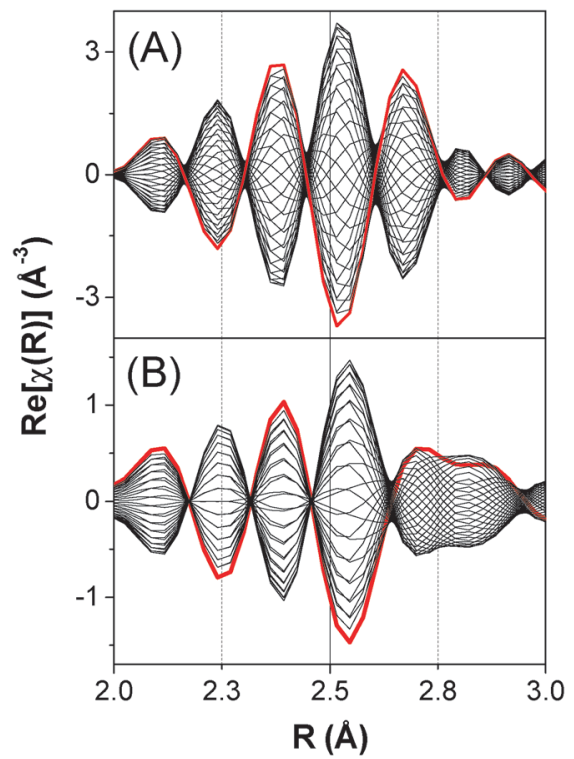

Fig. 7 Effect of phase lag on the real part $\operatorname{Re}[\chi(R)]$ of simulated phaseresolved FT-EXAFS spectra at the Pd K-edge $\left(0^{\circ}<\varphi^{\mathrm{PSD}}<360^{\circ}\right)$. (A) Effect of a phase lag within a single $\mathrm{Pd}-\mathrm{Pd}$ shell, where $N$ and $R$ varies from 12 and $2.7577 \AA$ to 6 and $2.8577 \AA$ with a phase lag $\phi_{\mathrm{N}}=0^{\circ}$ and $\phi_{\mathrm{R}}=40^{\circ}$. (B) Effect of a phase lag within two different and partially overlapping shells $(\mathrm{Pd}-\mathrm{Pd}$ and $\mathrm{Pd}-(\mathrm{O})-\mathrm{Pd})$, where only $N$ varies $\left(12<N_{\mathrm{Pd}-\mathrm{Pd}}<6\right.$ and $\left.0<N_{\mathrm{Pd}-(\mathrm{O})-\mathrm{Pd}}<4\right)$ with a phase lag $\phi_{\mathrm{N}, \mathrm{Pd}-\mathrm{Pd}}=0^{\circ}$ and $\phi_{\mathrm{N}, \mathrm{Pd}-(\mathrm{O})-\mathrm{Pd}}=40^{\circ}$.

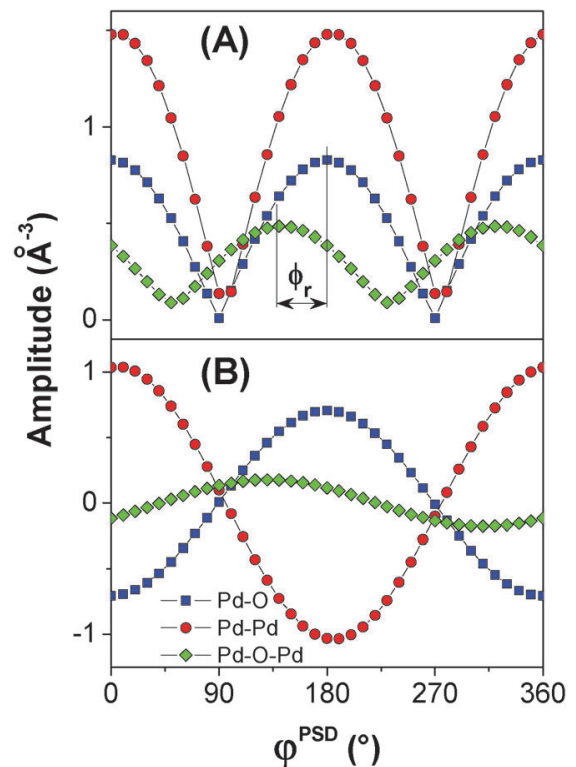

Fig. 8 (A) Variation of the amplitude of $|\chi(R)|$ and (B) of the real part $\operatorname{Re}[\chi(R)]$ of the simulated phase-resolved FT-EXAFS spectra as a function of the phase angle $\varphi^{\mathrm{PSD}}$ at the characteristic interatomic distances $R$ corresponding to the $\mathrm{Pd}-\mathrm{O}(\boldsymbol{\square}), \mathrm{Pd}-\mathrm{Pd}(\bullet)$, and $\mathrm{Pd}-(\mathrm{O})-\mathrm{Pd}(\bullet)$ shells. In (A) $R$ corresponds to $1.565 \AA, 2.512 \AA$ and $2.792 \AA$, respectively, whereas in (B) $R$ corresponds to $1.473 \AA$, $2.393 \AA$ and $2.976 \AA$, respectively.

shell can be hardly solved in our experimental time-resolved FT-EXAFS spectra of Fig. 10A because of the superimposition with the residual but dominant Pd-Pd shell. The ME approach coupled with PSD is a powerful tool to solve this issue thus

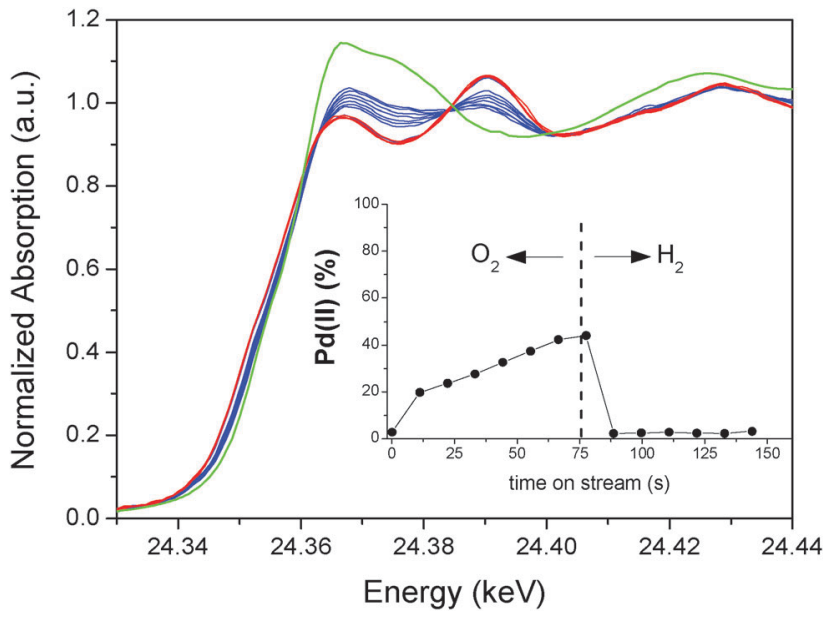

Fig. 9 Averaged time-resolved XANES spectra at the Pd K-edge of $1.6 \mathrm{wt} \%$ $\mathrm{Pd} / \mathrm{Al}_{2} \mathrm{O}_{3}$ recorded during a 1 vol\% $\mathrm{O}_{2} / \mathrm{He}$ (blue) to 1 vol\% $\mathrm{H}_{2} / \mathrm{He}$ (red) modulation experiment. The XANES spectrum of reference PdO is shown for comparison (green). Inset: temporal evolution of $\mathrm{Pd}(\|)$ obtained from the linear combination analysis of the XANES spectra of $\mathrm{PdO}$ and $\mathrm{Pd}$ foil.

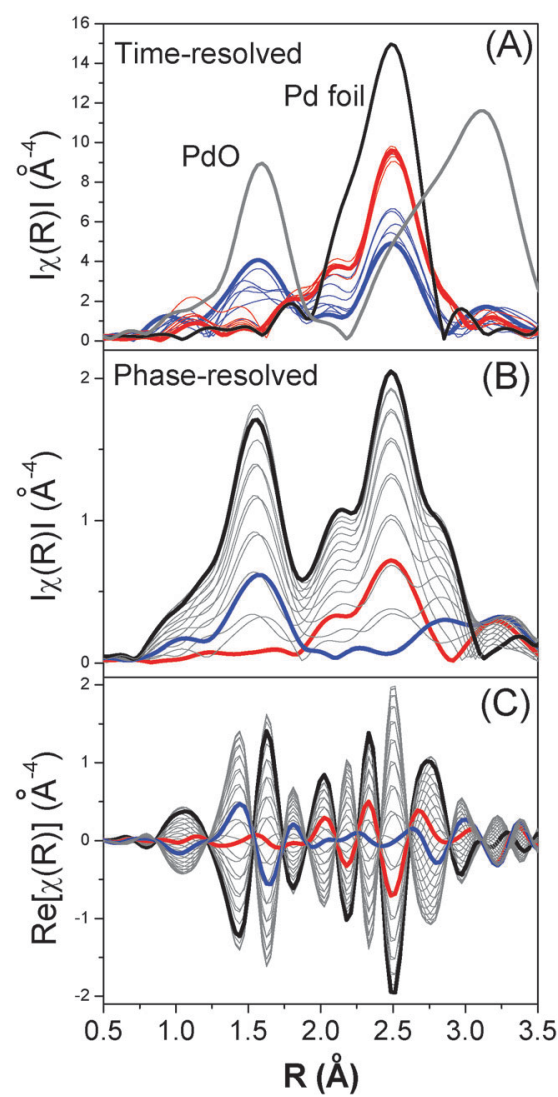

Fig. 10 ME-EXAFS results at the Pd K-edge obtained on $1.6 \mathrm{wt} \% \mathrm{Pd} / \mathrm{Al}_{2} \mathrm{O}_{3}$ during a 1 vol\% $\mathrm{O}_{2} / \mathrm{He}$ to $1 \mathrm{vol} \% \mathrm{H}_{2} / \mathrm{He}$ modulation experiment. (A) $k^{3}$-weighted FT-EXAFS spectra of bulk PdO (grey), Pd foil (black) and averaged time-resolved spectra recorded in 1 vol\% $\mathrm{O}_{2} / \mathrm{He}$ (blue) and in 1 vol\% $\mathrm{H}_{2} / \mathrm{He}$ (red); (B) phase-resolved FT-EXAFS spectra $\left(0^{\circ}<\varphi^{\text {PSD }}<\right.$ $\left.360^{\circ}\right)$; (C) real part of the phase-resolved FT-EXAFS spectra in (B). In (B) and (C) the spectra at $\varphi^{\mathrm{PSD}}=160^{\circ}$ (black line), $\varphi^{\mathrm{PSD}}=230^{\circ}$ (red line) and $\varphi^{\mathrm{PSD}}=$ $250^{\circ}$ (blue line) are highlighted. 
considerably improving the sensitivity of EXAFS spectroscopy as is described below.

The phase-resolved $k^{3}$-weighted $\chi(k)$ spectra (Fig. S12, ESI $\dagger$ ) exhibit a similar out of phase envelop to that of the simulated spectra of Fig. 3B, suggesting that some parameter possesses a different phase lag with respect to the others. Indeed, the Fourier transformation of the phase-resolved spectra (Fig. 10B) clearly shows the appearance of a peak at $2.82 \AA$. This signal is assigned to the $\mathrm{Pd}-(\mathrm{O})-\mathrm{Pd} 1$ shell and varies as a function of the phase angle similarly to the simulated spectra in the middle panel of Fig. 6D, the case of the $\mathrm{Pd}-(\mathrm{O})-\mathrm{Pd}$ shell having different phase lag compared to the Pd-Pd shell. This signal cannot be clearly distinguished in the time-resolved data of Fig. 10A, thus demonstrating the increased sensitivity of EXAFS by the use of PSD. The phase angular dependence of the three signals detected in the phase-resolved FT spectra at $1.56 \AA$ ( $\mathrm{Pd}-\mathrm{O}), 2.48 \AA(\mathrm{Pd}-\mathrm{Pd})$ and $2.82 \AA(\mathrm{Pd}-(\mathrm{O})-\mathrm{Pd} 1)$ with assignment of the negative values on the basis of the FT real part (Fig. 10C), according to the procedure previously illustrated in Fig. 8, demonstrates (Fig. 11) that the signal generated by the Pd-Pd shell varies in opposition and with a different phase lag $\phi_{\mathrm{r}}$ with respect to the other two shells. This behaviour reflects the red-ox dynamics of the Pd nano-particles, i.e. the periodic and alternate faster reduction than the re-oxidation, where the Pd-Pd shell decreases while the $\mathrm{Pd}-\mathrm{O}$ shell is forming. The different phase lag of the Pd-Pd shell represents a key finding because it allows us to isolate the contribution of the Pd-(O)-Pd shell from that of the residual metallic Pd in the EXAFS spectrum at $\varphi^{\mathrm{PSD}}=250^{\circ}$ (Fig. 11 and the blue curve in Fig. 10B). Similarly, the spectrum at $\varphi^{\mathrm{PSD}}=230^{\circ}$ contains the contribution of the variation of the Pd-Pd shell only, filtered from that of PdO (Fig. 11 and the red curve in Fig. 10B).

3.3.1 Quantitative analysis of experimental phase-resolved EXAFS spectra. An important consideration in the fitting procedure of phase-resolved spectra has to be mentioned at

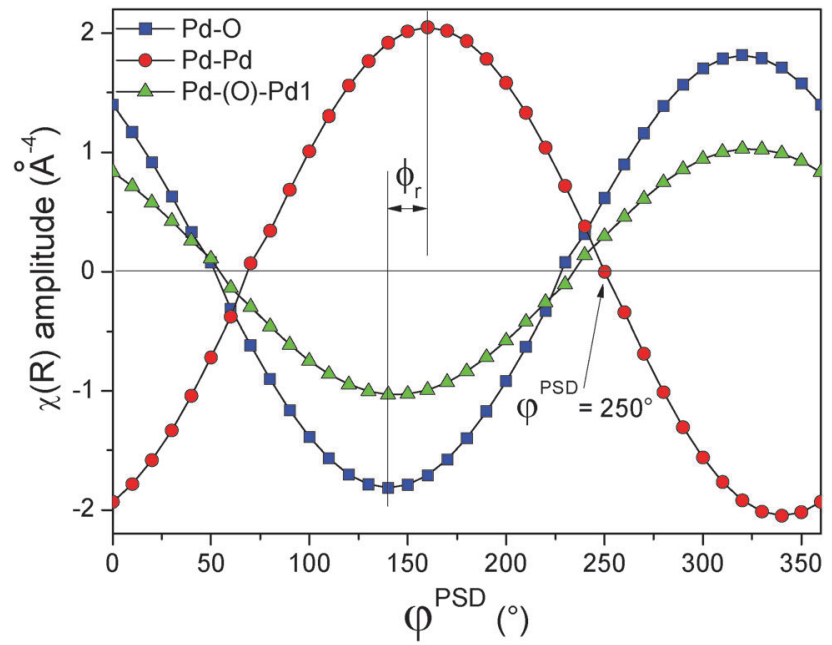

Fig. 11 Variation of the amplitude of the phase-resolved FT-EXAFS spectra as a function of the demodulation angle $\varphi^{\mathrm{PSD}}$ at bond distance $(R)$ of $1.565 \AA(\square), 2.823 \AA(\triangle)$ and $2.485 \AA(\bigcirc)$ corresponding to the $\mathrm{Pd}-\mathrm{O}$, $\mathrm{Pd}-(\mathrm{O})-\mathrm{Pd} 1$ and $\mathrm{Pd}-\mathrm{Pd}$ shells, respectively. The negative values of the amplitude have been assigned on the base of the variation of the corresponding FT real part. this point. We shall recall here that the demodulated spectrum is a difference spectrum between the potentially reversible oscillating states induced by the external stimulation. If a particular state appears and disappears during the stimulation, as it is in the case of PdO because the system oscillates between a reduced and a partially oxidized $\mathrm{Pd}$, then the isolated phaseresolved spectrum at a particular $\varphi^{\mathrm{PSD}}$ (in this case, 250 already contains the local structural information of that specific state, i.e. PdO. In this case, $\chi_{t}(k)_{\mathrm{i}}=0$ and it follows from eqn (4) that $\chi_{t}(k)_{\mathrm{f}}=A \chi_{\varphi}^{\mathrm{PSD}}(k)$, where $A$ is the scaling factor. Thus, this spectrum can be fitted by the conventional procedure to obtain the structural parameters $N, R$ and $\sigma^{2}$ after scaling the amplitude to the original value $\left(\Delta \chi_{k}\right)$ by applying eqn (4), i.e. multiplying $\chi_{\varphi}(k)$ by $2.1 / \cos \left(\phi-\varphi^{\mathrm{PSD}}\right)$ where $\varphi^{\mathrm{PSD}}=250^{\circ}$ for PdO in this case. The phase lag $\phi$ can be obtained by fitting the curve of Fig. 11 with eqn $(3)\left(\varphi_{\mathrm{Pd}-\mathrm{O}}^{\mathrm{PSD}}=321^{\circ}, \varphi_{\mathrm{Pd}-(\mathrm{O})-\mathrm{Pd} 1}^{\mathrm{PSD}}=324^{\circ}\right.$ and $\left.\varphi_{\mathrm{Pd}-\mathrm{Pd}}^{\mathrm{PSD}}=160^{\circ}\right)$. On the other hand, if a scattering shell varies along a modulation period but does not fully disappear, as it is in the case of the Pd-Pd shell, the phase-resolved spectrum contains information on the structural variation but does not represent the full structure, not even only one specific structure. In this case, the phase-resolved spectrum can be fitted as a difference spectrum as proposed by König et al. ${ }^{9}$ Alternately, it can be subtracted from the time-resolved $\chi(k)_{\mathrm{i}}$ spectrum according to eqn (4) in order to obtain the $\chi(k)_{\mathrm{f}}$ spectrum, which can then be fitted using the conventional EXAFS approach. This procedure is particularly effective in this example, where $\chi(k)_{\mathrm{i}}$ can be chosen as the last time-resolved spectrum recorded in $\mathrm{H}_{2}$ from which the scaled phase-resolved spectrum at $\varphi^{\mathrm{PSD}}=$ $230^{\circ}$ containing only the information about the Pd-Pd shell can be subtracted. The obtained $\chi(k)_{\mathrm{f}}$ spectrum has the advantage of containing a single coordination shell, thus filtered from the contribution of PdO. It can also be more easily fitted using fewer variables, thus increasing the consistency of the fit.

3.3.2 Fit results. As mentioned above, the structure of the initial state of the Pd nano-particle can be determined from the fitting of the last time-resolved spectrum recorded in $\mathrm{H}_{2}$. Because Pd at this stage is reduced, the $k^{3}$-weighted Fourier transformed EXAFS spectrum $\left(2<k<14 \AA^{-1}\right)$ has been fitted in the $R=0-3 \AA$ range considering a single Pd-Pd coordination shell (Fig. 12A). On the other hand, the structure of Pd in the final state, i.e. in $\mathrm{O}_{2}$, has been solved by fitting two extrapolated EXAFS spectra obtained from the phase-resolved ones containing the contribution of residual metallic Pd and of the newly formed $\mathrm{PdO}$, respectively. In particular, the structural parameters of PdO (i.e. $N, R$ and $\sigma^{2}$ of the $\mathrm{Pd}-\mathrm{O}, \mathrm{Pd}-(\mathrm{O})-\mathrm{Pd} 1$ and $\mathrm{Pd}-(\mathrm{O})-\mathrm{Pd} 2$ shells) have been obtained by the fitting of the phase-resolved EXAFS spectra at $\varphi^{\mathrm{PSD}}=250^{\circ}$ after properly scaling the amplitude as above. The structural parameters of the residual metallic Pd (i.e. $N, R$ and $\sigma^{2}$ of the Pd-Pd shell) were calculated by fitting the EXAFS spectrum obtained by subtracting the amplitude-scaled phase-resolved spectrum at $\varphi^{\mathrm{PSD}}=230^{\circ}$ from the last timeresolved EXAFS spectrum recorded under reducing conditions. The results of the fitting are presented in Table 1 and Fig. 12. The reduced Pd (Fig. 12A) possesses an average Pd-Pd coordination number $N$ of 11.4 that is typical of large nano-particles. ${ }^{28}$ 


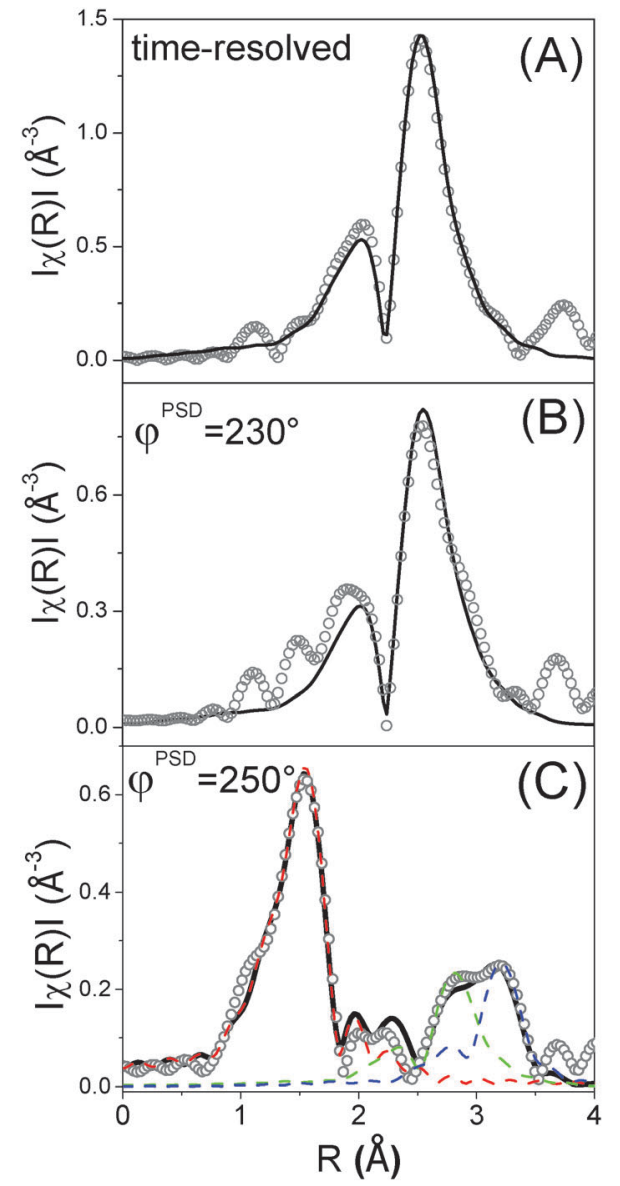

Fig. 12 Best fit of (A) the last time-resolved spectrum recorded in $\mathrm{H}_{2}$, (B) the spectrum in (A) subtracted by the amplitude-scaled phase-resolved $\chi(k)$ spectrum at $\varphi^{\mathrm{PSD}}=230^{\circ}$ and $(\mathrm{C})$ the amplitude-scaled phase-resolved $\chi(R)$ spectrum at $\varphi^{\mathrm{PSD}}=250^{\circ}$. Experimental data (open symbol) and best fit results (solid line). The contribution of the three fitted paths (dashed lines) $\mathrm{Pd}-\mathrm{O}(\mathrm{red}), \mathrm{Pd}-(\mathrm{O})-\mathrm{Pd} 1$ (green) and $\mathrm{Pd}-(\mathrm{O})-\mathrm{Pd} 2$ (blue) is shown in (C).

The interatomic distance of $2.794 \AA$ is slightly larger than the theoretical one $(2.758 \AA)$. This can be tentatively attributed the interstitial absorption of $\mathrm{H}$ atoms despite the relatively high temperature $(573 \mathrm{~K})$ and low hydrogen partial pressure (10 mbar). ${ }^{29,30}$ Wang et al. ${ }^{30}$ have recently studied the stability of Pd hydride up to $483 \mathrm{~K}$ and they have calculated a residual $\mathrm{H} /$ Pd ratio of 0.1 at this temperature for $2.1 \mathrm{~nm}$ Pd nanoparticle. $\sigma^{2}$ is relatively high $\left(0.012 \AA^{2}\right)$ due to the thermal disorder and probably the inhomogeneously dissolved $\mathrm{H}$ distribution. Under an $\mathrm{O}_{2}$ atmosphere both $R(2.790 \AA)$ and $N(7.7)$ of the Pd-Pd shell decrease (Fig. 12B), indicating a possible decrease of the particle size consequent to surface oxidation.

Fig. 12C demonstrates the most important finding of this work in agreement with the simulations shown above. The Fourier transform of the phase-resolved EXAFS spectrum at $\varphi^{\mathrm{PSD}}=250^{\circ}$ clearly displays the $\mathrm{Pd}-(\mathrm{O})-\mathrm{Pd} 1$ and $\mathrm{Pd}-(\mathrm{O})-\mathrm{Pd} 2$ shells which are otherwise hardly detectable in the timeresolved spectra. The contribution of the residual Pd-Pd shell has been removed by PSD. The fit of this spectrum provides the coordination number of the three $\mathrm{Pd}-\mathrm{O}, \mathrm{Pd}-(\mathrm{O})-\mathrm{Pd} 1$ and Pd-(O)-Pd2 shells of 2.0, 1.8 and 3.4, respectively (Table 1). These values are evidently much lower than those of the bulk PdO (4, 4 and 8, respectively), indicating that oxidation has not been extensive in agreement with our simulation. However, they are consistent with a PdO surface with two Pd atomic layers. For example, if the (001) surface of PdO is considered (Fig. 13) the average coordination number of the shells is 2, 4 and 4 , respectively, whereas for the (100) or the (010) surface it is 3,2 and 4, respectively. These values are lower for smaller particles because the atoms at the edge and corners exhibit lower coordination. It is important to note that the main difference between the (001) and the (100) surface of PdO is that the ratio between the coordination number of the $\mathrm{Pd}-(\mathrm{O})-$ $\mathrm{Pd} 2$ and $\mathrm{Pd}-(\mathrm{O})-\mathrm{Pd} 1$ is $1: 1$ in the former, whereas it is $2: 1$ in the latter. The fitting results suggest that, being the experimental Pd-(O)-Pd2: $\mathrm{Pd}-(\mathrm{O})-\mathrm{Pd} 1$ ratio of 1.9 , the (100) or the (010) surface are preferentially formed. ${ }^{31}$

\section{Conclusions}

This work demonstrates that the modulated excitation approach combined with phase sensitive analysis can be successfully applied to extended X-ray absorption fine structure spectroscopy despite the high number of variables involved. Several additional qualitative and quantitative information can be extrapolated

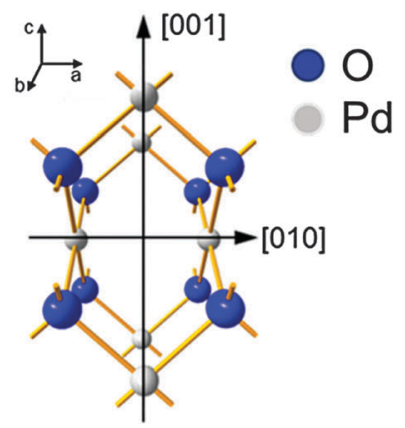

(A)

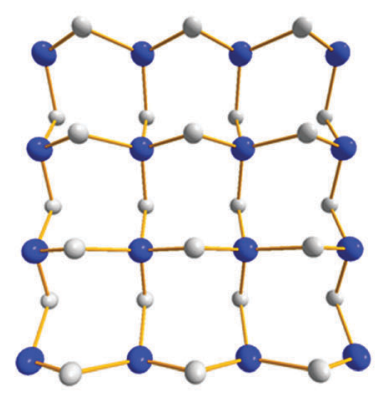

(B)

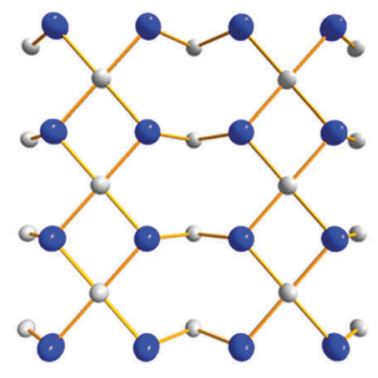

(C)

Fig. 13 (A) Tetragonal unit cell of PdO; (B) (001) $9.03 \AA \times 9.03 \AA$ PdO surface; (C) (100) or (010) $9.03 \AA \times 10.67 \AA$ PdO surface. 
from the phase-resolved FT-EXAFS spectra with respect to the original time-resolved set of data, thus significantly improving the sensitivity of EXAFS spectroscopy. A first inspection of the phase-resolved $\chi(k)$ spectra reveals whether one or more variables $\left(N, R\right.$ or $\left.\sigma^{2}\right)$, either within one shell or of a different shell, vary with a phase lag $\phi$ with respect to the other ones. The presence of a phase lag generates the typical out of phase envelop. The analysis of the real (or imaginary) part of the phase resolved FT-EXAFS spectra is crucial to recognize both (i) whether the phase lag occurs within one shell or in a different shell and (ii) to access the relative dynamics occurring during the modulation experiment, such as the formation/disappearance of a specific shell. The presence of one or more shells varying with a phase lag is a crucial point that allows extrapolation of the spectra of that specific shell/structure. One of the most important finding of this work is the possibility of isolating the contribution of the second $\mathrm{Pd}-(\mathrm{O})-\mathrm{Pd}$ shell of $\mathrm{PdO}$ otherwise hidden from the residual first $\mathrm{Pd}-\mathrm{Pd}$ shell of the metal Pd. This result could be in principle extended to any other minute structural change or intermediate species formed during a modulation experiment.

Because the phase resolved EXAFS spectra are scaled difference spectra with reduced noise two different approaches have been here proposed for the quantitative analysis of the corresponding FT spectra. In particular, the FT-phase-resolved spectrum can be directly fitted only if a shell fully appears and disappears during the modulation (e.g. the $\mathrm{Pd}-\mathrm{O}$ and $\mathrm{Pd}-(\mathrm{O})-\mathrm{Pd}$ shells of the experimental example). By contrast, if a shell does not fully disappear (e.g. the Pd-Pd shell) the spectrum of the final state (e.g. in $\mathrm{O}_{2}$ atmosphere) can be obtained by subtracting the selected demodulated $\chi_{\varphi}(k)$ from the time-resolved $\chi_{t}(k)_{\mathrm{i}}$ spectrum of the initial state $(e . g$. in $\mathrm{H}_{2}$ atmosphere) with the possible advantage of being isolated from the contribution of other structures.

Finally, the FT-EXAFS spectrum of PdO has been successfully isolated from that of the residual metallic Pd of partially oxidized Pd nanoparticles thanks to the application of PSD, thus allowing the fitting of the second $\mathrm{Pd}-(\mathrm{O})-\mathrm{Pd}$ shell with enhanced accuracy. This result has shown that an oxide layer covering a metallic core is formed under a fast switch from reducing to oxidizing atmosphere at $573 \mathrm{~K}$ on $1.6 \mathrm{wt} \% \mathrm{Pd} / \mathrm{Al}_{2} \mathrm{O}_{3}$.

Therefore, we have demonstrated the potential of the modulated excitation approach applied to quantitative EXAFS. The approach becomes powerful when minute structural changes of functional materials under relevant conditions need to be analyzed.

\section{List of symbols}

$\alpha$

$\delta_{i}(k)$

$\lambda(k)$

$\sigma_{i}^{2}$

$\phi$

$\varphi^{\mathrm{PSD}}$

$\chi_{t}(k)$
Amplitude correction factor

Photoelectron phase shift

Photoelectron mean free path

Debye-Waller factor

Phase lag between the stimulation and the system response

Demodulation phase angle

Time-resolved EXAFS spectrum
$\chi_{t}(R) \quad$ Fourier transform of the time-resolved EXAFS spectrum

$\chi_{\varphi}(k) \quad$ Phase-resolved EXAFS spectrum

$\chi_{\varphi}(R) \quad$ Fourier transform of the phase-resolved EXAFS spectrum

$|\chi(R)| \quad$ Magnitude of the Fourier transform EXAFS spectrum

$F_{i}(k) \quad$ Photoelectron scattering amplitude

$\operatorname{Im}[\chi(R)] \quad$ Imaginary part of the Fourier transform EXAFS spectrum

$K \quad$ Demodulation index

$k \quad$ Photoelectron wavenumber

$N_{i} \quad$ Coordination number

$\operatorname{Re}[\chi(R)] \quad$ Real part of the Fourier transform EXAFS spectrum

$R_{i} \quad$ Interatomic distance

$S_{0}{ }^{2} \quad$ Passive electron reduction factor

$T \quad$ Stimulation period

$W(k) \quad$ Window function

$\omega \quad$ Stimulation frequency

\section{Acknowledgements}

The authors kindly acknowledge the financial support from the Competence Centre for Materials Science and Technology (CCMX) through project No 66, the Swiss National Science Foundation under project No. 200021-138068 and the University of Milano through project No. 17777 "Piano di Sviluppo 2014 linea B1". The European Synchrotron Radiation Facility (ESRF, Grenoble, France) is acknowledged for providing the beamtime at beamline BM23. D.F. is grateful to Dr M. A. Newton for his precious and continued support and to Dr S. K. Matam, Dr S. Yoon and Dr Y. Lu for help during measurements.

\section{References}

1 S. R. Bare and T. Ressler, Adv. Catal., 2009, 52, 339-465.

2 P. Silveston, R. R. Hudgins and A. Renken, Catal. Today, 1995, 25, 91-112.

3 D. Baurecht and U. P. Fringeli, Rev. Sci. Instrum., 2001, 72, 3782-3792.

4 T. Bürgi and A. Baiker, J. Phys. Chem. B, 2002, 106, 10649-10658.

5 A. Urakawa, T. Bürgi and A. Baiker, Chem. Eng. Sci., 2008, 63, 4902-4909.

6 A. Eyssler, E. Kleymenov, A. Kupferschmid, M. Nachtegaal, S. K. Matam, P. Hug, A. Weidenkaff and D. Ferri, J. Phys. Chem. C, 2011, 115, 1231-1239.

7 D. Ferri, M. A. Newton and M. Nachtegaal, Top. Catal., 2011, 54, 1070-1078.

8 D. Ferri, S. K. Matam, R. Wirz, A. Eyssler, O. Korsak, P. Hug, A. Weidenkaff and M. A. Newton, Phys. Chem. Chem. Phys., 2010, 12, 5634-5646.

9 C. F. J. König, J. A. vanBokhoven, T. J. Schildhauer and M. Nachtegaal, J. Phys. Chem. C, 2012, 116, 19857-19866.

10 J. Stötzel, D. Lutzenkirchen-Hecht, R. Frahm, B. Kimmerle, A. Baiker, M. Nachtegaal, M. J. Beier and J. D. Grunwaldt, J. Phys.: Conf. Ser., 2009, 190, 012162. 
11 A. Haghofer, D. Ferri, K. Föttinger and G. Rupprechter, ACS Catal., 2012, 2, 2305-2315.

12 V. Marchionni, M. A. Newton, A. Kambolis, S. K. Matam, A. Weidenkaff and D. Ferri, Catal. Today, 2014, 229, 80-87.

13 G. L. Chiarello, M. Nachtegaal, V. Marchionni, L. Quaroni and D. Ferri, Rev. Sci. Instrum., 2014, 85, 074102.

14 D. E. Ramaker and D. C. Koningsberger, Phys. Chem. Chem. Phys., 2010, 12, 5514-5534.

15 C. S. Spanjers, T. P. Senftle, A. C. T. vanDuin, M. J. Janik, A. I. Frenkel and R. M. Rioux, Phys. Chem. Chem. Phys., 2014, 16, 26528-26538.

16 C. F. J. König, T. J. Schildhauer and M. Nachtegaal, J. Catal., 2013, 305, 92-100.

17 J. Stötzel, D. Lützenkirchen-Hecht, R. Frahm and J. D. Grunwaldt, J. Phys.: Conf. Ser., 2013, 430, 012126.

18 R. Caliandro, D. Chernyshov, H. Emerich, M. Milanesio, L. Palin, A. Urakawa, W. vanBeek and D. Viterbo, J. Appl. Crystallogr., 2012, 45, 458-470.

19 D. Chernyshov, W. vanBeek, H. Emerich, M. Milanesio, A. Urakawa, D. Viterbo, L. Palin and R. Caliandro, Acta Crystallogr., Sect. A: Found. Crystallogr., 2011, 67, 327-335.

20 W. vanBeek, H. Emerich, A. Urakawa, L. Palin, M. Milanesio, R. Caliandro, D. Viterbo and D. Chernyshov, J. Appl. Crystallogr., 2012, 45, 738-747.
21 D. C. Koningsberger and R. Prins, $X$-ray Absorption: principles, applications and techniques of EXAFS, SEXAFS and XANES, Wiley, 1988.

22 D. Ferri, M. A. Newton, M. DiMichiel, S. Yoon, G. L. Chiarello, V. Marchionni, S. K. Matam, M. H. Aguirre, A. Weidenkaff, F. Wen and J. Gieshoff, Phys. Chem. Chem. Phys., 2013, 15, 8629-8639.

23 M. A. Newton, Chem. Soc. Rev., 2008, 37, 2644-2657.

24 S. I. Zabinsky, J. J. Rehr, A. Ankudinov, R. C. Albers and M. J. Eller, Phys. Rev. [Sect.] B, 1995, 52, 2995-3009.

25 B. Ravel and M. Newville, J. Synchrotron Radiat., 2005, 12, 537-541.

26 M. A. Newton, Top. Catal., 2009, 52, 1410-1424.

27 S. K. Matam, E. H. Otal, M. H. Aguirre, A. Winkler, A. Ulrich, D. Rentsch, A. Weidenkaff and D. Ferri, Catal. Today, 2012, 184, 237-244.

28 A. Jentys, Phys. Chem. Chem. Phys., 1999, 1, 4059-4063.

29 A. L. Bugaev, A. A. Guda, K. A. Lomachenko, V. V. Srabionyan, L. A. Bugaev, A. V. Soldatov, C. Lamberti, V. P. Dmitriev and J. A. V. Bokhoven, J. Phys. Chem. C, 2014, 118, 10416.

30 J. Wang, Q. Wang, X. Jiang, Z. Liu, W. Yang and A. I. Frenkel, J. Phys. Chem. C, 2015, 119, 854.

31 D. T. Vu, K. A. R. Mitchell, O. L. Warren and P. A. Thiel, Surf. Sci., 1994, 318, 129-138. 